\title{
Swell induced surface instability of confined hydrogel layers on substrates
}

\author{
Min Kyoo Kang and Rui Huang* \\ Department of Aerospace Engineering and Engineering Mechanics, University of Texas, Austin, \\ $T X 78712$
}

\begin{abstract}
In response to external stimuli, polymeric hydrogels can change volume and shape dramatically. Experimental studies have observed a variety of instability patterns of hydrogels, due to swelling or shrinking, many of which have not been well understood. The present paper considers swell induced surface instability of a hydrogel layer on a rigid substrate. Based on a recently developed theoretical framework for neutral polymeric gels, a linear perturbation analysis is performed to predict the critical condition for onset of the surface instability. Using a nonlinear finite element method, numerical simulations are presented to show the swelling process, with evolution of initial surface perturbations followed by formation of crease-like surface patterns. In contrast to previously suggested critical conditions for surface creasing, the present study suggests a material specific condition that predicts a range of critical swelling ratios from about 2.5 to 3.4 and quantitatively relates the critical condition to material properties of the hydrogel system. A stability diagram is constructed with two distinct regions for stable and unstable hydrogels depending on two dimensionless material parameters.
\end{abstract}

Keywords: hydrogel, surface instability, creasing *ruihuang@mail.utexas.edu 


\section{Introduction}

Containing a high fraction of water, hydrogels are often found as structural materials and actuators in natural systems such as jelly fish and human tissues (Calvert, 2008). Synthetic gels are common ingredients in foods, cosmetics, and pharmaceuticals. Driven by typically large and reversible deformation of hydrogels in response to external stimuli (e.g., temperature, pH, electrical field), a wide range of applications have been demonstrated recently, including drug delivery (Peppas et al., 2006), tissue engineering (Uljin et al., 2007), micro-sensors and actuators (Beebe et al., 2000; Dong et al., 2006; Sidorenko et al., 2007).

Experimental studies have shown complex material behaviors of gels (Li and Tanaka, 1992; Tokarev and Minko, 2009). Subject to geometric confinement and/or mechanical constraint, a variety of instability patterns have been observed in gel-like materials (Southern and Thomas, 1965; Tanaka et al., 1987; Matsuo and Tanaka, 1992; Tirumala et al., 2005; Mora and Boudaoud, 2006; Sultan and Boudaoud, 2008; Trujillo et al., 2008; Zhang et al., 2008). Of particular interest, surface wrinkling of swollen rubber vulcanizates was observed by Southern and Thomas (1965), who reported a critical swelling ratio of about 2.5 due to the effect of substrate constraint. Later, a wide range of critical swelling ratios were observed for different gel systems, between 2.46 and 3.72 by H. Tanaka et al. (1992) and around 2 by Trujillo et al. (2008). T. Tanaka et al. (1987) found that many gels formed surface patterns during swelling process, and they suggested a critical osmotic pressure for the surface instability, although their analysis implied a critical compressive stress. More recently, Trujillo et al. (2008) showed that the critical condition for surface creasing in their experiments with a model hydrogel system agreed well with the prediction by a linear perturbation analysis for rubber under equi-biaxial compression (Biot, 1963), with a critical linear compressive strain $~ 33 \%$ relative to the state of free swelling 
for unconstrained hydrogels. On the other hand, Gent and Cho (1999) found that Biot's prediction considerably overestimated the critical strain for surface creasing in their experiments with rubbers compressed by mechanical bending. A recent work by Hong et al. (2009B) argued that surface creasing is a different mode of surface instability in contrast with Biot's linear perturbation analysis, and they predicted a critical swelling ratio at 2.4 for surface creasing of gels based on an energetic consideration and numerical calculations for neo-Hookean elastomers. It remains elusive how the critical swelling ratio varies from around 2 to 3.72 in experiments. In the present study, we extend Biot's linear perturbation analysis to swelling deformation of a hydrogel layer confined by a rigid substrate. The predicted critical swelling ratio varies from about 2.5 to 3.4, depending on the material parameters of the hydrogel. Using a nonlinear finite element method, we show by numerical simulations that an initial surface perturbation can evolve to form surface creases as a post-instability phenomenon.

The remainder of this paper is organized as follows. Section 2 briefly reviews a recently developed nonlinear theory for polymeric gels. Section 3 presents an analytical solution for homogeneous swelling of hydrogel layers laterally constrained by a rigid substrate. A linear perturbation analysis is performed in Section 4, which leads to an eigenvalue problem and predicts the critical condition for onset of surface instability. Section 5 presents numerical simulations with a nonlinear finite element method, showing formation of surface creases. Concluding remarks are given in Section 6.

\section{A nonlinear theory for hydrogels}

Consider a hydrogel immersed in a solvent (Fig. 1). A nonlinear theory has been developed previously for swelling deformation of neutral polymer gels (Hong et al., 2008 and 
2009A; Kang and Huang, 2009). Regardless of the transport kinetics, deformation of the hydrogel eventually reaches an equilibrium state when both the chemical potential and the mechanical stress satisfy the equilibrium condition. The chemical equilibrium requires that the chemical potential inside the gel be a constant and equal to the chemical potential of the external solvent $(\mu=\hat{\mu})$. In addition, the mechanical equilibrium requires that

$$
\frac{\partial s_{i J}}{\partial X_{J}}+B_{i}=0 \quad \text { in } \Omega_{0}
$$

and

$$
s_{i J} N_{J}=T_{i} \text { or } \delta x_{i}=0 \quad \text { on } \Gamma_{0},
$$

where $s_{i J}$ is the nominal stress, $B_{i}$ and $T_{i}$ are the nominal body force and surface traction, $\Omega_{0}$ and $\Gamma_{0}$ are the volume and the surface of the hydrogel at the reference state, $N_{J}$ is the unit vector in the outward normal direction of the surface, $X_{J}$ and $x_{i}$ are the particle coordinates at the reference and the current states, respectively, and they are related to each other through the deformation gradient tensor, $F_{i J}=d x_{i} / d X_{J}$. For the present study, the reference state is taken to be the undeformed dry state of the polymer network, and the body force is considered negligible.

The chemical potential of the external solvent $(\hat{\mu})$ in general depends on the temperature $(T)$ and pressure $(p)$. Assuming an ideal gas phase $\left(p<p_{0}\right)$ and an incompressible liquid phase $\left(p>p_{0}\right)$ for the solvent, the chemical potential is given by

$$
\hat{\mu}(p, T)=\left\{\begin{array}{cl}
\left(p-p_{0}\right) v, & \text { if } p>p_{0} \\
k_{B} T \ln \left(p / p_{0}\right), & \text { if } p<p_{0}
\end{array}\right.
$$

where $p_{0}$ is the equilibrium vapor pressure and depends on the temperature, $v$ is the volume per solvent molecule, and $k_{B}$ is the Boltzmann constant. At the equilibrium vapor pressure ( $p=p_{0}$ ), the external chemical potential $\hat{\mu}=0$. For water at $25^{\circ} \mathrm{C}, \quad p_{0} \sim 3.2 \mathrm{kPa}$ and $v \sim 3 \times 10^{-29} \mathrm{~m}^{3}$, the 
chemical potential is about $0.0007 k_{B} T$ under the atmospheric pressure ( $\left.101 \mathrm{kPa}\right)$. In a vacuum $(p=0), \hat{\mu}=-\infty$.

The constitutive behavior of the hydrogel can be described by using a free energy function $U$, which in general depends on both the elastic deformation of the polymer network and the concentration of solvent molecules inside the gel. The nominal stress in the gel is then

$$
S_{i J}=\frac{\partial U}{\partial F_{i J}} .
$$

To be specific, we adopt a free energy density function that consists of two separate parts, one for elastic deformation of polymer network and the other for mixing of solvent molecules with the polymer, namely

$$
U(\mathbf{F}, C)=U_{e}(\mathbf{F})+U_{m}(C) .
$$

where

$$
\begin{aligned}
& U_{e}(\mathbf{F})=\frac{1}{2} N k_{B} T(I-3-2 \ln J), \\
& U_{m}(C)=\frac{k_{B} T}{v}\left(v C \ln \frac{v C}{1+v C}+\frac{\chi v C}{1+v C}\right),
\end{aligned}
$$

and $I=F_{i J} F_{i J}, J=\operatorname{det}(\mathbf{F}), C$ is the nominal concentration (number per volume) of solvent molecules, $N$ is the effective number of polymer chains per unit volume of the hydrogel at the dry state, and $\chi$ is a dimensionless quantity characterizing the interaction energy between the solvent molecules and the polymer. Therefore, the material properties of the hydrogel system is fully determined by three parameters: $N k_{B} T, k_{B} T / v$, and $\chi$, the first of which is simply the initial shear modulus of the polymer network.

The elastic free energy density function $\left(U_{e}\right)$ in Eq. (2.6) is similar to that obtained by Flory (1953) based on a statistical mechanics model, but is slightly different as suggested in the 
previous works (Hong et al., 2008; Kang and Huang, 2009). The free energy of mixing $\left(U_{m}\right)$ in Eq. (2.7) is derived exactly from the Flory-Huggins polymer solution theory (Huggins, 1941; Flory, 1953), with the assumption of molecular incompressibility inside the gel, namely, the total volume of the hydrogel is the sum of the volume of the dry polymer network and the solvent molecules so that

$$
J=1+v C .
$$

At the equilibrium state of swelling, the chemical potential inside the hydrogel is a constant while the concentration field can be inhomogeneous. Thus, it is convenient to write the free energy function in terms of the chemical potential via a Legendre transformation (Hong et al., 2009A), namely

$$
\hat{U}(\mathbf{F}, \hat{\mu})=U(\mathbf{F}, C)-\hat{\mu} C .
$$

It then follows that, by Eqs. (2.4)-(2.8)

$$
s_{i J}=\frac{\partial \hat{U}}{\partial F_{i J}}=N k_{B} T\left(F_{i J}+\alpha H_{i J}\right),
$$

where

$$
\begin{aligned}
& \alpha=-\frac{1}{J}+\frac{1}{N \nu}\left(\ln \frac{J-1}{J}+\frac{1}{J}+\frac{\chi}{J^{2}}-\frac{\hat{\mu}}{k_{B} T}\right), \\
& H_{i J}=\frac{\partial J}{\partial F_{i J}}=\frac{1}{2} e_{i j k} e_{J K L} F_{j K} F_{k L} .
\end{aligned}
$$

\section{Homogeneous swelling of a confined hydrogel layer}

Now consider a hydrogel layer attached to a rigid substrate (Fig. 2a). Due to the confinement of the substrate, the hydrogel layer swells only in the thickness direction. Let 1 and 3 denote the in-plane directions and 2 the out-of-plane (thickness) direction. For 
homogeneous swelling, the deformation gradient tensor is diagonal, with the principal stretches, $F_{22}=\lambda_{h}$ and $F_{11}=F_{33}=1$. The volume swelling ratio is simply, $J_{h}=\lambda_{h}$, and the nominal concentration of solvent molecule in the hydrogel is

$$
C_{h}=\frac{\lambda_{h}-1}{v} .
$$

Upon swelling, the free energy density inside the hydrogel becomes

$$
U_{h}\left(\lambda_{h}\right)=U_{e}\left(\lambda_{h}\right)+U_{m}\left(C_{h}\right),
$$

where, by Eqs. (2.6) and (2.7),

$$
\begin{aligned}
& U_{e}\left(\lambda_{h}\right)=\frac{1}{2} N k_{B} T\left[\lambda_{h}^{2}-1-2 \ln \left(\lambda_{h}\right)\right], \\
& U_{m}\left(C_{h}\right)=\frac{k_{B} T}{v}\left[\left(\lambda_{h}-1\right) \ln \left(1-\frac{1}{\lambda_{h}}\right)+\chi\left(1-\frac{1}{\lambda_{h}}\right)\right] .
\end{aligned}
$$

The total free energy of the system (including the hydrogel and the external solvent) consists of the internal free energy and the chemical/mechanical work done during absorption and swelling, namely

$$
G=U_{h} V_{0}-\hat{\mu} C_{h} V_{0}+\left(J_{h}-1\right) p V_{0}=\left[U_{h}\left(\lambda_{h}\right)-\frac{\hat{\mu}-p v}{v}\left(\lambda_{h}-1\right)\right] V_{0},
$$

where $V_{0}$ is the reference volume of the layer at the dry state. Note that the external solvent exerts a pressure $p$ onto the surface of the hydrogel layer, which does a negative work and thus increases the free energy as the hydrogel swells.

The equilibrium swelling ratio of the hydrogel can then be determined by minimizing the total free energy. Setting $d G / d \lambda_{h}=0$ leads to

$$
\ln \left(1-\frac{1}{\lambda_{h}}\right)+\frac{1}{\lambda_{h}}+\frac{\chi}{\lambda_{h}^{2}}+N v\left(\lambda_{h}-\frac{1}{\lambda_{h}}\right)=\frac{\hat{\mu}-p v}{k_{B} T} .
$$


Solving Eq. (3.6) gives the homogeneous swelling ratio $\lambda_{h}$ for a specific hydrogel system as a function of the external chemical potential $(\hat{\mu})$, which in turn is a function of the temperature and pressure. By the definition of the chemical potential in Eq. (2.3), we note that, if the external solvent is in a liquid phase (i.e., $p>p_{0}$ ), the right hand side of Eq. (3.6) is independent of the pressure $p$ and thus the homogeneous swelling ratio depends on the temperature only, while this is not the case if $p<p_{0}$ (a gas phase). In the previous studies (Hong et al., 2009A; Kang and Huang, 2009), a similar solution was obtained by using a Lagrange multiplier, but the effect of the external pressure was ignored. Figure 3 plots the homogeneous swelling ratio as a function of the chemical potential, comparing the present solution to the previous solution. In addition to the two dimensionless material parameters ( $N v$ and $\chi)$ for the hydrogel, the present solution depends on the normalized equilibrium vapor pressure, $\bar{p}_{0}=p_{0} v /\left(k_{B} T\right)$, which is a function of the temperature for a specific solvent. At a constant temperature, the hydrogel swells increasingly as the chemical potential increases until the pressure reaches the equilibrium vapor pressure ( $p=p_{0}$ and $\mu=0$ ). For a typical value of the equilibrium vapor pressure (e.g., for water at $25^{\circ} \mathrm{C}, \quad \bar{p}_{0} \sim 2.3 \times 10^{-5}$ ), the difference between the two solutions is negligible when $\mu \leq 0$ or $p \leq p_{0}$. However, when $p>p_{0}$, the previous solution predicts that the swelling ratio continues to increase as the chemical potential or pressure increases, while the present solution predicts a constant swelling ratio independent of the chemical potential or pressure. Consequently, given the material parameters for a hydrogel system ( $N v$ and $\chi)$, the maximum degree of homogeneous swelling occurs at the equilibrium vapor pressure $(\mu=0)$. Also plotted in Fig. 3 is the homogeneous swelling ratio for an artificially large equilibrium vapor pressure $\left(\bar{p}_{0}=0.01\right)$, to illustrate the effect of solvent vapor pressure on the swelling ratio, i.e., $\lambda_{h}$ decreases as $\bar{p}_{0}$ 
increases. Similar effect can be predicted for swelling of unconstrained hydrogels.

The lateral confinement by the substrate induces a compressive stress in the swollen hydrogel layer. By Eq. (2.10) and Eq. (3.6), we obtain that

$$
s_{i J}=N k_{B} T\left(F_{i J}-\lambda_{h} H_{i J}\right)-p H_{i J} .
$$

It then follows that

$$
s_{11}=s_{33}=s_{h}=-N k_{B} T\left(\lambda_{h}^{2}-1\right)-p \lambda_{h},
$$

and $s_{22}=-p$, while all other stress components are zero. The solution for the nominal stress differs slightly from the previous studies (Hong et al., 2009A; Kang and Huang, 2009) due to the effect of the external pressure.

The true (Cauchy) stresses at the current state are related to the nominal stresses as $\sigma_{11}=\sigma_{33}=s_{h} / \lambda_{h}=-N k_{B} T\left(\lambda_{h}-1 / \lambda_{h}\right)-p$ and $\sigma_{22}=s_{22}=-p$. The hydrostatic pressure inside the hydrogel layer is thus

$$
p_{i n}=-\frac{1}{3}\left(\sigma_{11}+\sigma_{22}+\sigma_{33}\right)=\frac{2}{3} N k_{B} T\left(\lambda_{h}-\frac{1}{\lambda_{h}}\right)+p .
$$

The difference between the internal pressure of the gel and the external pressure of the solvent defines an osmotic pressure, namely

$$
p_{o s}=p_{i n}-p=\frac{2}{3} N k_{B} T\left(\lambda_{h}-\frac{1}{\lambda_{h}}\right) .
$$

\section{Linear perturbation analysis}

To examine stability of the homogeneous swelling deformation of the confined hydrogel layer, we assume a small perturbation with displacements from the swollen state in both the thickness and lateral directions (Fig. 2b), namely 


$$
u_{1}=u_{1}\left(x_{1}, x_{2}\right) \text { and } u_{2}=u_{2}\left(x_{1}, x_{2}\right)
$$

In the spirit of linear perturbation analysis similar to that by Biot (1963), along with the transverse isotropy of the homogeneous solution, the two-dimensional perturbation is sufficient to represent an arbitrary perturbation in three dimensions.

The deformation gradient after the perturbation becomes

$$
\widetilde{\mathbf{F}}=\left[\begin{array}{ccc}
1+\frac{\partial u_{1}}{\partial x_{1}} & \lambda_{h} \frac{\partial u_{1}}{\partial x_{2}} & 0 \\
\frac{\partial u_{2}}{\partial x_{1}} & \lambda_{h}\left(1+\frac{\partial u_{2}}{\partial x_{2}}\right) & 0 \\
0 & 0 & 1
\end{array}\right]
$$

Thus, the volume ratio of swelling (relative to the dry state) is

$$
J=\operatorname{det}(\widetilde{\mathbf{F}}) \approx \lambda_{h}\left(1+\frac{\partial u_{1}}{\partial x_{1}}+\frac{\partial u_{2}}{\partial x_{2}}\right)=\lambda_{h}(1+\varepsilon),
$$

where $\varepsilon=\frac{\partial u_{1}}{\partial x_{1}}+\frac{\partial u_{2}}{\partial x_{2}}$ is the linear volumetric strain relative to the homogeneously swollen state, and only the first-order terms of the perturbation are retained for the linear analysis. As a result, the concentration field in the hydrogel becomes inhomogeneous, namely

$$
v C=J-1 \approx v C_{h}+\lambda_{h} \varepsilon
$$

By substituting Eq. (4.2) into Eq. (2.10), we obtain the nominal stresses after the perturbation

$$
s_{i J} \approx N k_{B} T\left[\tilde{F}_{i J}-\left(\lambda_{h}-\xi_{h} \varepsilon\right) \tilde{H}_{i J}\right]-p \tilde{H}_{i J}
$$

where

$$
\xi_{h}=\frac{1}{\lambda_{h}}+\frac{1}{N v}\left(\frac{1}{\lambda_{h}-1}-\frac{1}{\lambda_{h}}-\frac{2 \chi}{\lambda_{h}^{2}}\right)
$$


Expanding Eq. (4.5) gives the stress components explicitly as follows:

$$
\begin{aligned}
& s_{11} \approx s_{h}+N k_{B} T\left[\left(1+\lambda_{h} \xi_{h}\right) \frac{\partial u_{1}}{\partial x_{1}}+\lambda_{h}\left(\xi_{h}-\lambda_{h}\right) \frac{\partial u_{2}}{\partial x_{2}}\right]-p \lambda_{h} \frac{\partial u_{2}}{\partial x_{2}}, \\
& s_{22} \approx-p+N k_{B} T\left[\left(\xi_{h}-\lambda_{h}\right) \frac{\partial u_{1}}{\partial x_{1}}+\left(\xi_{h}+\lambda_{h}\right) \frac{\partial u_{2}}{\partial x_{2}}\right]-p \frac{\partial u_{1}}{\partial x_{1}}, \\
& s_{33} \approx s_{h}+N k_{B} T \lambda_{h}\left(\xi_{h}-\lambda_{h}\right) \varepsilon-p \lambda_{h} \varepsilon \\
& s_{12} \approx N k_{B} T \lambda_{h}\left(\frac{\partial u_{1}}{\partial x_{2}}+\frac{\partial u_{2}}{\partial x_{1}}\right)+p \frac{\partial u_{2}}{\partial x_{1}}, \\
& s_{21} \approx N k_{B} T\left(\lambda_{h}^{2} \frac{\partial u_{1}}{\partial x_{2}}+\frac{\partial u_{2}}{\partial x_{1}}\right)+p \lambda_{h} \frac{\partial u_{1}}{\partial x_{2}}
\end{aligned}
$$

and $s_{23}=s_{32}=s_{13}=s_{31}=0$. Apparently, the linearized stress-strain relationship for the hydrogel layer becomes anisotropic due to the anisotropic swelling deformation before perturbation.

By substituting the stress components into the equilibrium equations (2.1) and setting the body force $B_{i}=0$, we obtain that

$$
\begin{aligned}
& \left(1+\lambda_{h} \xi_{h}\right) \frac{\partial^{2} u_{1}}{\partial x_{1}^{2}}+\lambda_{h}^{2} \frac{\partial^{2} u_{1}}{\partial x_{2}^{2}}+\lambda_{h} \xi_{h} \frac{\partial^{2} u_{2}}{\partial x_{1} \partial x_{2}}=0 \\
& \frac{\partial^{2} u_{2}}{\partial x_{1}^{2}}+\lambda_{h}\left(\xi_{h}+\lambda_{h}\right) \frac{\partial^{2} u_{2}}{\partial x_{2}^{2}}+\lambda_{h} \xi_{h} \frac{\partial^{2} u_{1}}{\partial x_{1} \partial x_{2}}=0
\end{aligned}
$$

Applying Fourier transform with respect to $x_{1}$ in Eqs. (4.12) and (4.13), we obtain that

$$
\begin{aligned}
& -k^{2}\left(1+\lambda_{h} \xi_{h}\right) \hat{u}_{1}+\lambda_{h}^{2} \frac{d^{2} \hat{u}_{1}}{d x_{2}^{2}}+i k \lambda_{h} \xi_{h} \frac{d \hat{u}_{2}}{d x_{2}}=0, \\
& -k^{2} \hat{u}_{2}+\lambda_{h}\left(\xi_{h}+\lambda_{h}\right) \frac{d^{2} \hat{u}_{2}}{d x_{2}^{2}}+i k \lambda_{h} \xi_{h} \frac{d \hat{u}_{1}}{d x_{2}}=0,
\end{aligned}
$$

where $i=\sqrt{-1}, \hat{u}_{1}\left(x_{2} ; k\right)$ and $\hat{u}_{2}\left(x_{2} ; k\right)$ are the Fourier transforms of $u_{1}\left(x_{1}, x_{2}\right)$ and 
$u_{2}\left(x_{1}, x_{2}\right)$ with the wave number $k$ in the $x_{1}$ direction.

The general solution to Eqs. (4.14) and (4.15) takes the form

$$
\hat{u}_{1}=\bar{u}_{1} \exp \left(q x_{2}\right) \text { and } \quad \hat{u}_{2}=\bar{u}_{2} \exp \left(q x_{2}\right)
$$

Substitution of (4.16) into Eqs. (4.14) and (4.15) leads to an eigenvalue problem

$$
\begin{aligned}
& {\left[-k^{2}\left(1+\lambda_{h} \xi_{h}\right)+\lambda_{h}^{2} q^{2}\right] \bar{u}_{1}+i k \lambda_{h} \xi_{h} q \bar{u}_{2}=0,} \\
& i k \lambda_{h} \xi_{h} q \bar{u}_{1}+\left[-k^{2}+\lambda_{h}\left(\xi_{h}+\lambda_{h}\right) q^{2}\right] \bar{u}_{2}=0,
\end{aligned}
$$

for which the eigenvalues are solved from the characteristic equation

$$
\left|\begin{array}{cc}
-k^{2}\left(1+\lambda_{h} \xi_{h}\right)+\lambda_{h}^{2} q^{2} & i k \lambda_{h} \xi_{h} q \\
i k \lambda_{h} \xi_{h} q & -k^{2}+\lambda_{h}\left(\xi_{h}+\lambda_{h}\right) q^{2}
\end{array}\right|=0
$$

or equivalently,

$$
\left(\lambda_{h}+\xi_{h}\right) \lambda_{h}^{3} q^{4}-\left(\xi_{h}+2 \lambda_{h}+\lambda_{h}^{2} \xi_{h}\right) \lambda_{h} k^{2} q^{2}+\left(1+\lambda_{h} \xi_{h}\right) k^{4}=0
$$

Solving Eq. (4.20), we obtain four eigenvalues

$$
q_{1,2}= \pm \frac{k}{\lambda_{h}} \text { and } q_{3,4}= \pm k \beta
$$

where $\beta=\sqrt{\frac{1+\lambda_{h} \xi_{h}}{\lambda_{h}^{2}+\lambda_{h} \xi_{h}}}$. There exist two sets of degenerated solutions to the eigenvalue problem, when $\xi_{h}=0$ or $\lambda_{h} \xi_{h}=-1$, as given in the Appendix. They have no substantial effect on the stability analysis that follows.

For each eigenvalue, $q_{n}(n=1-4)$, an eigenvector, $\left(\bar{u}_{1}^{(n)}, \bar{u}_{2}^{(n)}\right)$, is obtained from Eqs. (4.17) and (4.18). Therefore, the complete solution to Eqs. (4.14) and (4.15) are

$$
\hat{u}_{1}\left(x_{2} ; k\right)=\sum_{n=1}^{4} A_{n} \bar{u}_{1}^{(n)} \exp \left(q_{n} x_{2}\right)
$$




$$
\hat{u}_{2}\left(x_{2} ; k\right)=\sum_{n=1}^{4} A_{n} \bar{u}_{2}^{(n)} \exp \left(q_{n} x_{2}\right)
$$

where, except for the degenerated cases,

$$
\left[\begin{array}{llll}
\bar{u}_{1}^{(1)} & \bar{u}_{1}^{(2)} & \bar{u}_{1}^{(3)} & \bar{u}_{1}^{(4)} \\
\bar{u}_{2}^{(1)} & \bar{u}_{2}^{(2)} & \bar{u}_{2}^{(3)} & \bar{u}_{2}^{(4)}
\end{array}\right]=\left[\begin{array}{cccc}
1 & 1 & 1 & 1 \\
-i \lambda_{h} & i \lambda_{h} & -i \beta & i \beta
\end{array}\right] .
$$

The perturbation displacement can then be obtained by inverse Fourier transform of Eqs. (4.22) and (4.23), which may be considered as superposition of many Fourier components. For each Fourier component with a specific wave number $k$, the displacement is periodic in the $x_{1}$ direction, but varies exponentially in the $x_{2}$ direction for each eigen mode, similar to Biot's analysis for surface instability of a half-space rubber-like medium under compression (Biot, 1963), but in contrast with the sinusoidal variation assumed by Tanaka et al. (1987).

For each wave number $k$, the amplitudes for the four eigen modes $\left(A_{n}, n=1-4\right)$ are obtained by applying the boundary conditions. The lower surface of the hydrogel layer is attached to the rigid substrate with zero displacement, i.e.,

$$
u_{1}=u_{2}=0 \quad \text { at } x_{2}=0 \text {. }
$$

The upper surface of the hydrogel is subjected to a normal traction due to the pressure of external solvent. To the first order of perturbation, the nominal magnitude of the traction at the perturbed state is

$$
T \approx p\left(1+\frac{\partial u_{1}}{\partial x_{1}}\right)
$$

and the direction is perpendicular to the perturbed surface with the unit vector,

$$
n_{1} \approx \frac{\partial u_{2}}{\partial x_{1}} \text { and } n_{2} \approx-1+\frac{1}{2}\left(\frac{\partial u_{2}}{\partial x_{1}}\right)^{2}
$$

Therefore, by Eq. (2.2) and to the first order of perturbation, the traction boundary condition at 
the upper surface is

$$
s_{12}=p \frac{\partial u_{2}}{\partial x_{1}} \text { and } s_{22}=-p\left(1+\frac{\partial u_{1}}{\partial x_{1}}\right) \text { at } x_{2}=h
$$

where $h=\lambda_{h} h_{0}$ is the thickness of the hydrogel layer at the swollen state (before perturbation) and $h_{0}$ is the dry-state thickness.

Substituting Eqs. (4.22) and (4.23) into Eqs. (4.8) and (4.10) and then applying the boundary conditions in (4.25) and (4.28), we obtain that

$$
\begin{aligned}
& \sum_{n}^{4} A_{n} \bar{u}_{1}^{(n)}=0, \\
& \sum_{n}^{4} A_{n} \bar{u}_{2}^{(n)}=0, \\
& \sum_{n=1}^{4}\left[i k\left(\xi_{h}-\lambda_{h}\right) \bar{u}_{1}^{(n)}+\left(\xi_{h}+\lambda_{h}\right) q_{n} \bar{u}_{2}^{(n)}\right] A_{n} \exp \left(q_{n} h\right)=0, \\
& \sum_{n}^{4}\left[\lambda_{h} q_{n} \bar{u}_{1}^{(n)}+i k \lambda_{h} \bar{u}_{2}^{(n)}\right] A_{n} \exp \left(q_{n} h\right)=0 .
\end{aligned}
$$

Re-write Eqs. (4.26)-(4.29) in a matrix form as

$$
\sum_{n=1}^{4} D_{m n}(k) A_{n}=0
$$

where the coefficient matrix is given by

$$
\left[D_{m n}\right]=\left[\begin{array}{cccc}
1 & 1 & 1 & 1 \\
-\lambda_{h} & \lambda_{h} & -\beta & \beta \\
2 \lambda_{h} e^{k h_{0}} & 2 \lambda_{h} e^{-k h_{0}} & \left(\lambda_{h}+\frac{1}{\lambda_{h}}\right) e^{\beta k h} & \left(\lambda_{h}+\frac{1}{\lambda_{h}}\right) e^{-\beta k h} \\
\left(\lambda_{h}+\frac{1}{\lambda_{h}}\right) e^{k h_{0}} & -\left(\lambda_{h}+\frac{1}{\lambda_{h}}\right) e^{-k h_{0}} & 2 \beta e^{\beta k h} & -2 \beta e^{-\beta k h}
\end{array}\right] .
$$

The homogeneous swelling deformation of the hydrogel layer becomes unstable when 
Eq. (4.33) yields nontrivial solutions for the amplitudes, $A_{n}(n=1-4)$. The critical condition for onset of the instability is thus predicted by setting the determinant of the matrix $\mathbf{D}$ to be zero, namely

$$
\operatorname{det}\left[D_{m n}\right]=f\left(k h_{0}, \lambda_{h} ; N v, \chi\right)=0
$$

For each $k h_{0}$, Eq. (4.35) predicts a critical swelling ratio, $\lambda_{c}\left(k h_{0} ; N v, \chi\right)$, which depends on the perturbation wave number $k h_{0}$ as well as the two material parameters ( $N v$ and $\left.\chi\right)$ of the hydrogel. The corresponding critical chemical potential $\left(\mu_{c}\right)$ can then be determined from the homogeneous solution, Eq. (3.6).

Figure $4 \mathrm{a}$ plots the predicted critical swelling ratio as a function of $k h_{0}$, and Figure $4 \mathrm{~b}$ plots the critical chemical potential, for $\bar{p}_{0}=2.3 \times 10^{-5}, N v=0.001$, and different values of $\chi$. Unlike the critical compression for surface instability of a semi-infinite rubber, which is independent of the perturbation wavelength (Biot, 1963), the critical swelling ratio for swell induced surface instability of a hydrogel layer depends on the normalized perturbation wave number, $k h_{0}$, due to the presence of a rigid substrate. The substrate confinement tends to stabilize long-wavelength perturbations (with small $k h_{0}$ ), while the confinement effect diminishes for short-wavelength perturbations (with large $k h_{0}$ ). Consequently, the critical swelling ratio decreases as $k h_{0}$ increases and approaches a constant at the limit of short-wavelength perturbations $\left(k h_{0} \rightarrow \infty\right)$. Therefore, the onset of swell-induced surface instability is controlled by the minimum critical swelling ratio at the short-wavelength limit. It is speculated that surface effects (e.g., surface energy, surface stress), while not considered in the present study, could potentially stabilize short-wavelength perturbations and, together with the substrate confinement effect, could lead to an intermediate wavelength for onset of the surface instability.

We note in Fig. 4 that, for $\chi \leq 0.6$, there exists a critical wave number, for which the 
critical swelling ratio equals the maximum homogeneous swelling ratio and the corresponding critical chemical potential equals zero. For smaller perturbation wave numbers, the hydrogel layer remains stable at the equilibrium chemical potential $(\mu=\hat{\mu}=0)$. For $\chi>0.6$, however, we find that the hydrogel layer remains stable for all possible perturbation wave numbers; thus no critical condition is predicted. As shown later (Fig. 6), for each $N v$, there exists a critical value for $\chi$, beyond which the hydrogel layer is stable and swells homogeneously at the equilibrium state.

Next we focus our attention on the critical condition at the short-wavelength limit. By letting $k h_{0} \rightarrow \infty$ in Eq. (4.34) and setting the determinant of the matrix to be zero, we obtain that

$$
f_{\infty}\left(\lambda_{h} ; N v, \chi\right)=\left[\left(\lambda_{h}+\frac{1}{\lambda_{h}}\right)^{2}-4 \lambda_{h} \beta\right]\left(\beta-\lambda_{h}\right)=0 .
$$

It can be shown that $\beta-\lambda_{h} \neq 0$ for all swelling hydrogels $\left(\lambda_{h}>1\right)$. Thus, the critical condition becomes

$$
\left(\lambda_{h}+\frac{1}{\lambda_{h}}\right)^{2}-4 \lambda_{h} \beta=0
$$

Combining this with the definition of $\beta$ in (4.21) and $\xi_{h}$ in (4.6) gives a nonlinear equation, which can be solved to predict the critical swelling ratio at the short-wavelength limit, $\lambda_{c}^{\infty}(N v, \chi)$. The corresponding critical chemical potential, $\mu_{c}^{\infty}\left(N v, \chi, \bar{p}_{0}\right)$, is calculated from Eq. (3.6) by setting $\lambda_{h}=\lambda_{c}^{\infty}(N v, \chi)$.

Figure 5a plots the predicted critical swelling ratio $\left(\lambda_{c}^{\infty}\right)$ as a function of $N v$ for different values of $\chi$, and Fig. $5 \mathrm{~b}$ plots the critical chemical potential $\left(\mu_{c}^{\infty}\right)$, assuming a constant equilibrium vapor pressure $\left(\bar{p}_{0}=2.3 \times 10^{-5}\right)$. The dashed lines in Fig. 5a show the maximum 
homogeneous swelling ratio predicted by Eq. (3.6). For each $\chi$, the critical chemical potential increases monotonically with increasing $N v$ until it reaches the equilibrium chemical potential $\left(\mu_{c}^{\infty}=0\right.$ ), at which point the critical swelling ratio equals the maximum homogeneous swell ratio. Therefore, the stability of the homogeneously swollen hydrogel layer depends on both $N v$ and $\chi$. The two dimensionless material parameters characterize the elastic stiffness of the polymer network and the polymer-solvent interaction, respectively. While the polymer stiffness increases with $N v$, the network tends to swell more significantly in a good solvent (low $\chi$ ) than in a poor solvent (high $\chi$ ). The interplay between the two parameters is summarized in a diagram (Fig. 6) with two distinct regions for stable and unstable hydrogels. The boundary line separating the two regions is determined by setting $\bar{\mu}_{c}^{\infty}=0$ or $\lambda_{c}^{\infty}(N v, \chi)=\max \left(\lambda_{h}\right)$ in Eq. (4.37). The range of $N v$ in Fig. 6 roughly corresponds to a range between $1 \mathrm{kPa}$ and $10 \mathrm{MPa}$ for the initial shear modulus $\left(N k_{B} T\right)$ of the polymer network at $25^{\circ} \mathrm{C}$, which is typical for hydrogels and elastomers. For a hydrogel layer with properties in the upper-right region of the diagram (stiff network, poor solvent), it swells homogeneously and remains stable at the equilibrium chemical potential ( $\mu=0)$. The homogeneous swelling ratio is typically small $\left(\lambda_{h}<3\right)$ in this region. For a hydrogel layer with properties in the lower-left region (soft network, good solvent), surface instability occurs at a critical swelling ratio (Fig. 5a) before it reaches the maximum homogeneous swelling ratio. The predicted critical swelling ratio, ranging between 2.5 and 3.4, depends on both $N v$ and $\chi$.

We note that, when $N v$ is relatively small $\left(<10^{-4}\right)$, the critical swelling ratio (Fig. $5 \mathrm{a}$ ) is nearly a constant ( 3.4) independent of $N v$ or $\chi$, and the critical value of $\chi$ that separates the unstable and stable regions in Fig. 6 is nearly independent of $N v$. On the other hand, the critical 
chemical potential (Fig. 5b), nearly independent of $N v$, increases with increasing $\chi$. These results may be understood intuitively by considering the limiting case when the contribution of elasticity is negligible $(N v \rightarrow 0)$ for both the homogeneous swelling and the stability analysis. In this case, the competition between the entropy of mixing and the enthalpy of solvent-polymer interaction dominates the swelling process. Consequently, the stability of the hydrogel layer depends on $\chi$ only. As $N v \rightarrow 0, \xi_{h} \rightarrow \infty$ and $\beta \rightarrow 1$. Solving Eq. (4.37) gives a nontrivial solution for the constant critical swelling ratio, $\lambda_{c}=3.38$. By Eq. (3.6), the critical chemical potential is approximately

$$
\frac{\mu_{c}}{k_{B} T} \approx 0.0875 \chi-0.0549
$$

Setting $\mu_{c}=0$ in Eq. (4.38), we obtain that $\chi_{c}=0.63$. This critical value of $\chi$ is slightly higher than the critical value that was used to define a good solvent $(\chi<0.5)$ for swelling polymer networks (Li and Tanaka, 1992).

Previously, Tanaka et al. (1987) suggested a critical osmotic pressure above which distinct surface instability patterns appear in swelling gel slabs. As defined in Eq. (3.10), the osmotic pressure can be determined from the homogeneous swelling ratio. However, their theoretical analysis assumed inhomogeneous swelling of the gel slab even before the onset of instability and the predicted critical pressure depends on variation of the elastic modulus at the swollen state, which cannot be readily evaluated for quantitative comparisons. It has also been suggested that the compressive stress developed in the swollen hydrogel is the driving force for surface instability of confined hydrogel layers (Trujillo et al., 2008). Based on the homogeneous solution in Section 3, the critical compressive stress is obtained as 


$$
\sigma_{c}=N k_{B} T\left(\lambda_{c}-\frac{1}{\lambda_{c}}\right)+p_{0} \exp \left(\frac{\mu_{c}}{k_{B} T}\right)
$$

Using the critical swelling ratio and the critical chemical potential in Fig. 5, we plot the critical compressive stress as a function of $N v$ in Fig. 7a. For comparison, the dashed lines show the compressive stress at the maximum homogeneous swelling ratio. Again, the critical compressive stress in general depends on both $N v$ and $\chi$. When $N v<10^{-4}$, we have approximately,

$$
\sigma_{c} \approx N k_{B} T\left[3.1+\frac{\bar{p}_{0}}{N v} \exp (0.0875 \chi-0.0549)\right],
$$

which weakly depends on $\chi$. Interestingly, the first term in the bracket of Eq. (4.40) compares closely with Biot's prediction of the critical stress $\left(\sigma_{c}=3.08 N k_{B} T\right)$ for an incompressible rubber-like half-space under plane-strain compression (Biot, 1963). The second term becomes significant when the value of $N v$ is comparable to or smaller than the normalized vapor pressure $\left(\bar{p}_{0}=2.3 \times 10^{-5}\right)$.

Based on their experiments with a model system of poly(acrylamide-co-sodium acrylate) hydrogels, Trujillo et al. (2008) found that the onset of surface creasing instability corresponds to an effective linear compressive strain of $\sim 0.33$, in close agreement with Biot's prediction for a rubber-like half-space under equi-biaxial compression. We calculate the effective linear strain by comparing the laterally confined hydrogel layer to un-constrained free swelling of the same hydrogel system. As given in the previous studies (Hong et al., 2009A; Kang and Huang, 2009), the linear swelling ratio for free swelling can be obtained as a function of the chemical potential by solving the following equation 


$$
\ln \left(1-\frac{1}{\lambda_{0}^{3}}\right)+\frac{1}{\lambda_{0}^{3}}+\frac{\chi}{\lambda_{0}^{6}}+N v\left(\frac{1}{\lambda_{0}}-\frac{1}{\lambda_{0}^{3}}\right)=\frac{\hat{\mu}-p v}{k_{B} T}
$$

Similar to Eq. (3.6), we have modified the previous solution slightly to account for the external pressure $(p)$ such that the free swelling ratio $\left(\lambda_{0}\right)$ is independent of the external chemical potential when $\hat{\mu}>0$ or $p>p_{0}$. The effective linear strain from the state of free swelling to that of the laterally confined swelling is then

$$
\varepsilon=\frac{\lambda_{0}-1}{\lambda_{0}}
$$

Figure 7b plots the effective linear strain corresponding to the critical chemical potential in Fig. 5b, where the dashed lines show the maximum strain at $\mu=0$. While the maximum strain decreases monotonically with increasing $N v$, the critical strain for swell-induced surface instability increases with $N v$, due to the increasing critical chemical potential. Remarkably, the predicted critical strain is very close to 0.33 for hydrogel systems with $N v<10^{-4}$. By using the approximate solution for the critical chemical potential in Eq. (4.38), we obtain approximately, $\lambda_{0} \approx 0.0875^{-1 / 6}=1.5$, and thus $\varepsilon \approx 0.33$

Among various critical quantities shown in Figs. 5 and 7, the critical swelling ratio can be directly measured experimentally. As noticed previously (Hong et al., 2009B), the wide range of the reported critical swelling ratios (from 2 to 3.72) has not been well understood. While the present study predicts a range of critical swelling ratios (from 2.5 to 3.4), in reasonable agreement with the reported values, quantitative comparisons for specific hydrogel systems are not possible at the moment, because the two key parameters $(N v$ and $\chi)$ that determine the critical swelling ratio in the present model are not readily available from the reported experiments. In principle, both $N v$ and $\chi$ can be measured by independent experiments. 
For example, $N v$ is related to the initial shear modulus of the polymer network $\left(N k_{B} T\right)$, which in turn can be related to the crosslink density and molecular weight (Treloar, 1975). The interaction parameter $\chi$ can be determined by measuring the volume ratio of free swelling (e.g., Gee, 1946). It was also suggested that $\chi$ is inversely proportional to the temperature ( $\mathrm{Li}$ and Tanaka, 1992). Consequently, the stability of the confined hydrogel layer may depend on temperature.

We close this section by pointing out that the present analysis of surface instability assumes a quasi-statically controlled swelling process, where the chemical potential is ramped up slowly as a loading parameter and the hydrogel swells to an equilibrium state at each loading step until the onset of surface instability. The same process will be simulated numerically using a nonlinear finite element method in the next section. However, in many experiments (e.g., Tanaka et al., 1987; Trujillo et al., 2008), as a hydrogel is immersed in a solvent of a constant chemical potential (like a step loading), swelling is a kinetic process with non-equilibrium transient states. The kinetics of molecular transport coupled with large deformation of the polymer network could lead to a rich dynamics of evolving surface instability patterns, which presents an interesting topic for future studies.

\section{Numerical simulations}

In this section, we use a nonlinear finite element method developed in a previous study (Kang and Huang, 2009) to numerically simulate swelling of a confined hydrogel layer and surface evolution beyond the critical point predicted by the linear perturbation analysis. The hydrogel layer is modeled with two-dimensional plane-strain elements (CPE4) in the commercial package ABAQUS (2008), along with a self-developed user subroutine (UMAT) for the 
constitutive behavior of hydrogels. The lower surface of the hydrogel layer is fixed, while the upper surface is subjected to a pressure that depends on the chemical potential according to Eq. (2.3). Symmetric boundary conditions are imposed at the two vertical sidewalls of the model so that the layer can only swell in the thickness direction. The chemical potential in the hydrogel is ramped up as a loading parameter, and the equilibrium equations of the system are solved at each step by a nonlinear solver based on the Newton-Raphson method. As in the previous study (Kang and Huang, 2009), to circumvent the numerical difficulty with the negative infinite chemical potential at the dry state, we start each numerical simulation with an initial state of homogeneous swelling, for which the chemical potential can be determined analytically by Eq. (3.6) for a specific hydrogel system. A small perturbation is then introduced as surface imperfection, which is generated using cubic spline curves in ABAQUS.

Figure 8(a-e) show the snapshots from one simulation, for a hydrogel layer with $N v=$ 0.001 and $\chi=0.4$. Figure 8a shows the initial state of homogeneous swelling $\left(\lambda_{h}=2\right.$ and $\bar{\mu}=-$ 0.0916) with a small surface perturbation. At $\bar{\mu}=-0.00456$ (Fig. 8b), the hydrogel layer has swollen nearly twice as much while the surface perturbation has grown considerably, resulting in a clearly inhomogeneous distribution of the compressive stress $\left(\sigma_{11}\right)$ in the layer. As the chemical potential continues to rise, the surface perturbation evolves to form localized grooves (Fig. 8c), and eventually the two sides of the groove fold into other, forming surface creases (Fig. 8, d and e). A frictionless, hard self-contact is defined for the surface to prevent penetration. The simulation is stopped at $\bar{\mu}=0$. The evolution of the hydrogel surface is shown more clearly in Fig. 9 (a-e), where a self-similar growth of the initial perturbation is followed by formation of surface grooves and creases. It is thus suggested that the onset of swell induced surface instability in a confined hydrogel layer as predicted by the linear perturbation analysis in Section 
4 could grow into surface creases as a result of the nonlinear post-instability effect. As an analogy, formation of surface grooves has been shown in simulations for surface evolution of stressed crystals facilitated by surface diffusion (Yang and Srolovitz, 1994; Pang and Huang, 2006), as a nonlinear phenomenon following the growth of initially linear perturbations.

\section{Concluding Remarks}

Based on a previously developed theoretical framework, we have presented a linear perturbation analysis for swelling deformation of a confined hydrogel layer on a rigid substrate, which provides a theoretical understanding on the critical condition for onset of surface instability. The predicted critical condition depends on the two dimensionless material parameters $(N v$ and $\chi)$ of specific hydrogel systems. In particular, the critical swelling ratio varies from 2.5 to 3.4. Using a nonlinear finite element method, numerical simulations are presented to show the swelling process of a confined hydrogel layer, with evolution of an initial surface perturbation followed by formation of surface creases.

As pointed out previously (Trujillo et al., 2008), the surface instability places a fundamental limit on the degree of swelling for a confined hydrogel layer without formation of undesirable surface features for applications such as cell culture and smart surface coatings. Here we suggest that such a limit shall be understood on a system specific basis, which also opens the possibilities to achieve an optimal degree of swelling by selecting a specific solvent system along with molecular structures of the polymer network. Furthermore, theoretical understanding on the critical condition and post-instability surface evolution could also facilitate development of controllable surface patterns in soft materials for a range of applications (e.g., microdevices and tissue engineering) (Guvendiren et al., 2009). The field is wide open as surface instability of 
hydrogels can be influenced by many other effects not considered in the present study, such as kinetics and electrochemistry in polyelectrolyte (PE) gels.

\section{ACKNOWLEDGMENTS}

The authors gratefully acknowledge financial support by National Science Foundation through Grant No. CMMI-0547409.

\section{APPENDIX}

For completeness, we present here the degenerated solutions to the eigenvalue problem in Eqs. (4.17)-(4.18). First, the eigenvalue problem is degenerated when

$$
\xi_{h}=\frac{1}{\lambda_{h}}+\frac{1}{N v}\left(\frac{1}{\lambda_{h}-1}-\frac{1}{\lambda_{h}}-\frac{2 \chi}{\lambda_{h}^{2}}\right)=0 .
$$

It can be shown that this is only possible when $\chi>0.5$. In this case, the two equilibrium equations in (4.12)-(4.13) become uncoupled, and the eigenvalue problem becomes

$$
\begin{aligned}
& {\left[-k^{2}+\lambda_{h}^{2} q^{2}\right] \bar{u}_{1}=0,} \\
& {\left[-k^{2}+\lambda_{h}^{2} q^{2}\right] \bar{u}_{2}=0,}
\end{aligned}
$$

which has two eigenvalues, $q_{1,2}= \pm \frac{k}{\lambda_{h}}$.

The complete solution then takes the form

$$
\hat{u}_{1}\left(x_{2} ; k\right)=A_{1} \exp \left(\frac{k x_{2}}{\lambda_{h}}\right)+A_{2} \exp \left(-\frac{k x_{2}}{\lambda_{h}}\right),
$$




$$
\hat{u}_{2}\left(x_{2} ; k\right)=A_{3} \exp \left(\frac{k x_{2}}{\lambda_{h}}\right)+A_{4} \exp \left(-\frac{k x_{2}}{\lambda_{h}}\right)
$$

Applying the boundary conditions in (4.25) and (4.28), we obtain Eq. (4.33), but with the coefficient matrix as follows:

$$
\left[D_{m n}\right]=\left[\begin{array}{cccc}
1 & 1 & 0 & 0 \\
0 & 0 & 1 & 1 \\
-i \lambda_{h} e^{k h_{0}} & -i \lambda_{h} e^{-k h_{0}} & e^{k h_{0}} & -e^{-k h_{0}} \\
e^{k h_{0}} & -e^{-k h_{0}} & i \lambda_{h} e^{k h_{0}} & i \lambda_{h} e^{-k h_{0}}
\end{array}\right]
$$

Setting the determinant of (A.6) to be zero, we have

$$
\lambda_{h}=\operatorname{coth}\left(k h_{0}\right)
$$

Eq. (A.7) gives the critical swelling ratio as a function of $k h_{0}$, but only for the degenerated cases when Eq. (A.1) is satisfied.

A second degeneration of the eigenvalue problem occurs when $\beta=\sqrt{\frac{1+\lambda_{h} \xi_{h}}{\lambda_{h}^{2}+\lambda_{h} \xi_{h}}}=0$, or equivalently, when $\lambda_{h} \xi_{h}=-1$. In this case, we have three eigenvalues instead of four in Eq. (4.21), namely

$$
q_{1,2}= \pm \frac{k}{\lambda_{h}} \text { and } q_{3}=q_{4}=0
$$

Consequently, the complete solution becomes

$$
\begin{aligned}
& \hat{u}_{1}\left(x_{2} ; k\right)=\sum_{n=1}^{2} A_{n} \bar{u}_{1}^{(n)} \exp \left(q_{n} x_{2}\right)+A_{3}+A_{4} k x_{2}, \\
& \hat{u}_{2}\left(x_{2} ; k\right)=\sum_{n=1}^{2} A_{n} \bar{u}_{2}^{(n)} \exp \left(q_{n} x_{2}\right)-i A_{4},
\end{aligned}
$$

where the eigen vectors for the first two eigenvalues are the same as in Eq. (4.24). Again, applying the boundary conditions in (4.25) and (4.28), we obtain Eq. (4.33), but with the 
coefficient matrix as follows:

$$
\left[D_{m n}\right]=\left[\begin{array}{cccc}
1 & 1 & 1 & 0 \\
-\lambda_{h} & \lambda_{h} & 0 & -1 \\
2 \lambda_{h} e^{k h_{0}} & 2 \lambda_{h} e^{-k h_{0}} & \left(\lambda_{h}+\frac{1}{\lambda_{h}}\right) & \left(\lambda_{h}^{2}+1\right) k h_{0} \\
\left(\lambda_{h}+\frac{1}{\lambda_{h}}\right) e^{k h_{0}} & -\left(\lambda_{h}+\frac{1}{\lambda_{h}}\right) e^{-k h_{0}} & 0 & 2
\end{array}\right] .
$$

Setting the determinant of (A.11) gives the critical swelling ratio for the second degenerated case. It is found that, for all material parameters considered in the present study, the critical swelling ratios for both the degenerated cases are greater than the maximum homogeneous swelling ratio at the equilibrium chemical potential. Therefore, the prediction of swell induced surface instability is unaffected by the degeneration in the eigenvalue problem. 


\section{REFERENCES}

ABAQUS (version 6.8), 2008. Dassault Systèmes Simulia Corp., Providence, RI, USA.

Beebe, D. J., Moore, J. S., Bauer, J. M., Yu, Q., Liu, R. H., Devadoss, C., Jo, B.-H., 2000. Functional hydrogel structures for autonomous flow control inside microfluidic channels. Nature 404, 588-590.

Biot, M.A., 1963. Surface instability of rubber in compression. Appl. Sci. Res. A 12, 168-182.

Calvert, P., 2008. Gel sensors and actuators. Mat. Res. Soc. Bulletin 33, 207-212.

Dong. L., Agarwal, A. K., Beebe., D. J., Jiang, H., 2006. Adaptive liquid microlenses activated by stimuli-responsive hydrogels. Nature, 442, 551-554.

Flory, P.J., 1953. Principles of Polymer Chemistry, Cornell University Press. Ithaca. NY.

Gee, G., 1946. The interaction between rubber and liquids. IX. The elastic behaviour of dry and swollen rubbers. Trans. Faraday Soc. 42, 585-598.

Gent, A. N. and Cho, I. S., 1999. Surface instabilities in compressed or bent rubber blocks. Rubber Chemistry and Technology 72, 253-262.

Guvendiren, M., Yang, S., Burdick, J.A., 2009. Swelling-induced surface patterns in hydrogels with gradient crosslinking density. Advanced Functional Materials 19, 3038-3045.

Hong, W., Liu, Z., Suo, Z., 2009A. Inhomogeneous swelling of a gel in equilibrium with a solvent and mechanical load. Int. J. Solids Struct. 46, 3282-3289.

Hong, W., Zhao, X., Suo, Z., 2009B. Formation of creases on the surfaces of elastomers and gels. Appl. Phys. Lett. 95, 111901.

Hong, W., Zhao, X., Zhou, J., Suo, Z., 2008. A theory of coupled diffusion and large deformation in polymeric gels. J. Mech. Phys. Solids 56, 1779-1793.

Huggins, M.L., 1941. Solutions of long chain compounds. J. Chem. Phys. 9, 440.

Kang, M. K., Huang, R., 2009. A variational approach and finite element implementation for swelling of polymeric hydrogels under geometric constraints. J. Appl. Mech., accepted for publication. Preprint available at http://www.ae.utexas.edu/ ruihuang/papers/hydrogel2009.pdf. 
Li, Y., Tanaka, T., 1992. Phase transitions of gels. Annu. Rev. Mater. Sci., 22, 243-277.

Matsuo, E. S., Tanaka, T., 1992, Patterns in shrinking gels, Nature 358, 482-485.

Mora, T., Boudaoud, A., 2006. Buckling of swelling gels. European Phys. J. E 20, 119-124.

Pang, Y.Y., Huang, R., 2006. Nonlinear effect of stress and wetting on surface evolution in epitaxial thin films. Phys. Rev. B 74, 075413.

Peppas, N.A., Hilt, J.Z., Khademhosseini, A., Langer, R., 2006. Hydrogels in biology and medicine: From molecular principles to bionanotechnology. Advanced Materials 18, 1345-1360.

Sidorenko, A., Krupenkin, T., Taylor, A., Fratzl, P. Aizenberg, J., 2007. Reversible switching of hydrogel-actuated nanostructures into complex micropatterns. Science 315, 487-490.

Southern, E., Thomas, A.G., 1965. Effect of constraints on the equilibrium swelling of rubber vulcanizates. J. Polymer Science A 3, 641-646.

Sultan, E., Boudaoud, A., 2008. The buckling of a swollen thin gel layer bound to a compliant substrate. J. Appl. Mech. 75, 051002.

Tanaka, H., Tomita, H., Takasu, A., Hayashi, T., Nishi, T., 1992. Morphological and kinetic evolution of surface patterns in gels during the swelling process: evidence of dynamic pattern ordering. Phys. Rev. Lett. 68, 2794-2797.

Tanaka, T., Sun, S.-T., Hirokawa, Y., Katayama, S., Kucera, J., Hirose, Y., Amiya, T., 1987. Mechanical instability of gels at the phase transition. Nature 325, 796-198.

Tirumala, V. R., Divan, R., Ocola, L. E., Mancini, D. C., 2005. Direct-write e-beam patterning of stimuli-responsive hydrogel nanostructures. J. Vac. Sci. Technol. B 23, 3124-3128.

Tokarev, I, Minko, S., 2009. Stimuli-responsive hydrogel thin films. Soft Matter 5, 511-524.

Treloar, L.R.G., 1975. The Physics of Rubber Elasticity. Oxford University Press, Oxford.

Trujillo, V., Kim, J., Hayward, R. C., 2008. Creasing instability of surface-attached hydrogels. Soft Matter 4, 564-569.

Uljin, R. V., Bibi, N., Jayawarna, V., Thornton, P. D., Todd, S. J., Mart, R. J., Smith A. M., Gough, J. E., 2007. Bioresponsive hydrogels. Materials Today 10, 40-48.

Yang, W. H., Srolovitz, D.J., 1994. Surface morphology evolution in stressed solids: Surface diffusion controlled crack initiation. J. Mech. Phys. Solids 42, 1551-1574. 
Zhang, Y., Matsumoto, E. A., Peter, A., Lin, P.-C., Kamien, R. D., Yang, S., 2008. Onestep nanoscale assembly of complex structures via harnessing of an elastic instability. Nano Lett. 8, 1192-1196. 


\section{Figure Captions}

Figure 1. Schematic illustration of the reference state (dry state) and the equilibrium state of a hydrogel in a solvent with an chemical potential $\hat{\mu}$.

Figure 2. Schematic illustrations for (a) homogeneous swelling of a hydrogel layer, and (b) a perturbation to the swollen hydrogel layer.

Figure 3. Homogeneous swelling ratio of a hydrogel layer $(N v=0.001$ and $\chi=0.4)$ as a function of the external chemical potential.

Figure 4. (a) Critical swelling ratio and (b) the corresponding chemical potential, predicted by the linear perturbation analysis, versus the perturbation wave number for $N v=0.001$.

Figure 5. (a) Critical swelling ratio and (b) critical chemical potential, predicted by the linear perturbation analysis at an infinite wave number (short-wavelength limit), versus $N v$ for different values of $\chi$.

Figure 6. A stability diagram for substrate-confined hydrogel layers.

Figure 7. (a) Critical compressive stress and (b) critical linear strain, versus $N v$ for different values of $\chi$. The dashed lines in (a) show the compressive stress at the maximum homogeneous swelling, and the dashed lines in (b) show the effective strain for homogeneous swelling at $\mu=0$. The horizontal dash-dotted line in (b) indicates the critical strain (0.33) for a semi-infinite rubber under equi-biaxial compression.

Figure 8. Numerical simulation of swell-induced surface instability of a confined hydrogel layer $(N v=0.001$ and $\chi=0.4)$ on a rigid substrate. Contours show distribution of the compressive true stress in the lateral direction $\left(\sigma_{11}\right)$. (a) Initial perturbation at $\bar{\mu}=-0.0916$; (b) $\bar{\mu}=-0.00456$; (c) $\bar{\mu}=-0.00126$; (d) $\bar{\mu}=-0.000713$; and (e) $\bar{\mu}=0$. The stress magnitude in the scale bar is normalized by the initial shear modulus of the polymer network $\left(N k_{B} T\right)$.

Figure 9. Evolution of the surface profile of a confined hydrogel layer $(N v=0.001$ and $\chi=0.4)$ on a rigid substrate. (a) Initial perturbation at $\bar{\mu}=-0.0916$; (b) $\bar{\mu}=-0.00456$; (c) $\bar{\mu}=-$ 0.00126; (d) $\bar{\mu}=-0.000713$; and (e) $\bar{\mu}=0$. 

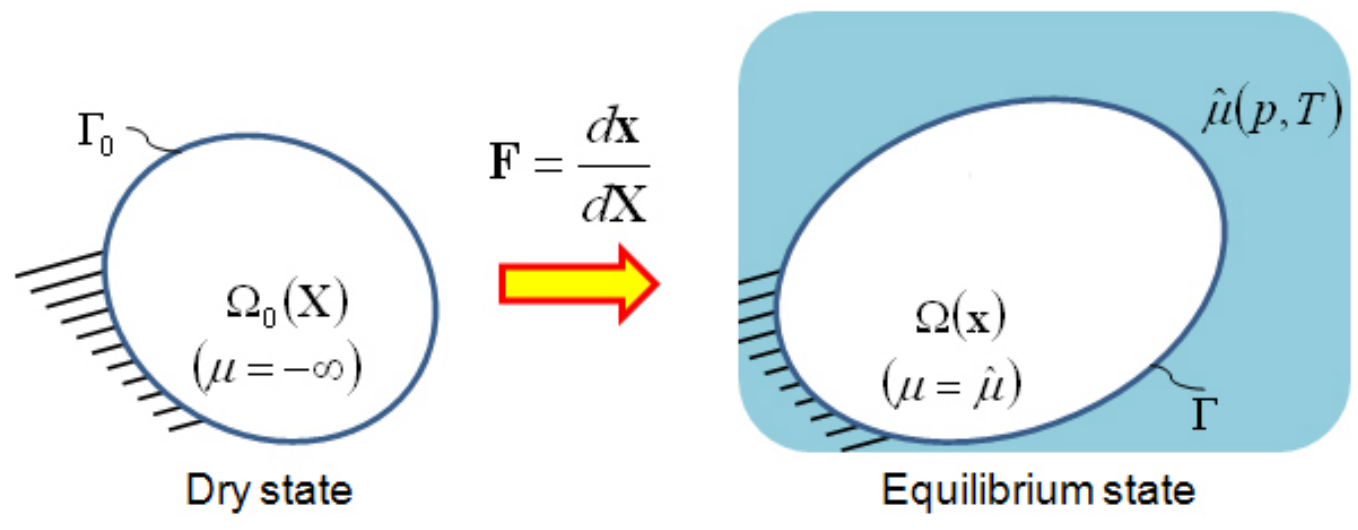

Figure 1. Schematic illustration of the reference state (dry state) and the equilibrium state of a hydrogel in a solvent with an chemical potential $\hat{\mu}$. 

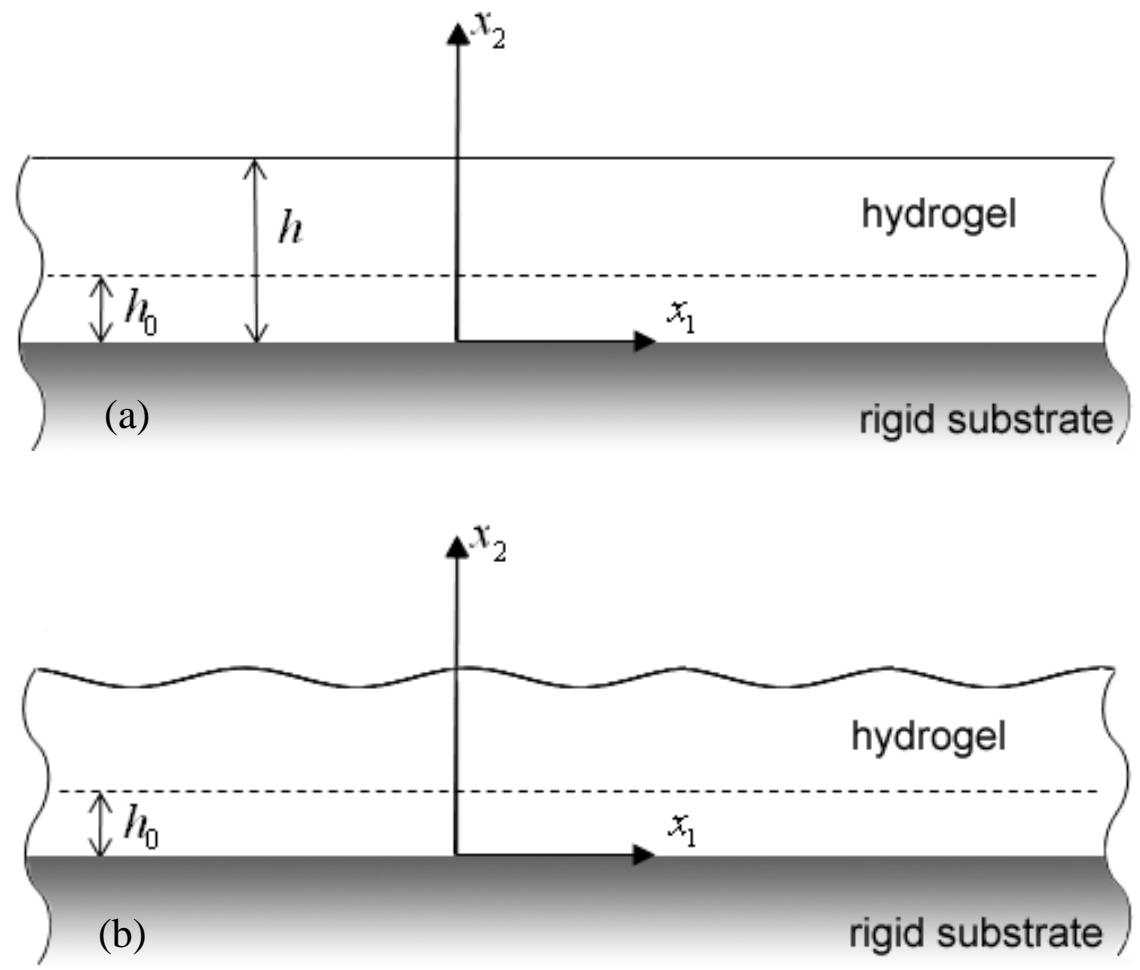

Figure 2. Schematic illustrations for (a) homogeneous swelling of a hydrogel layer, and (b) a perturbation to the swollen hydrogel layer. 


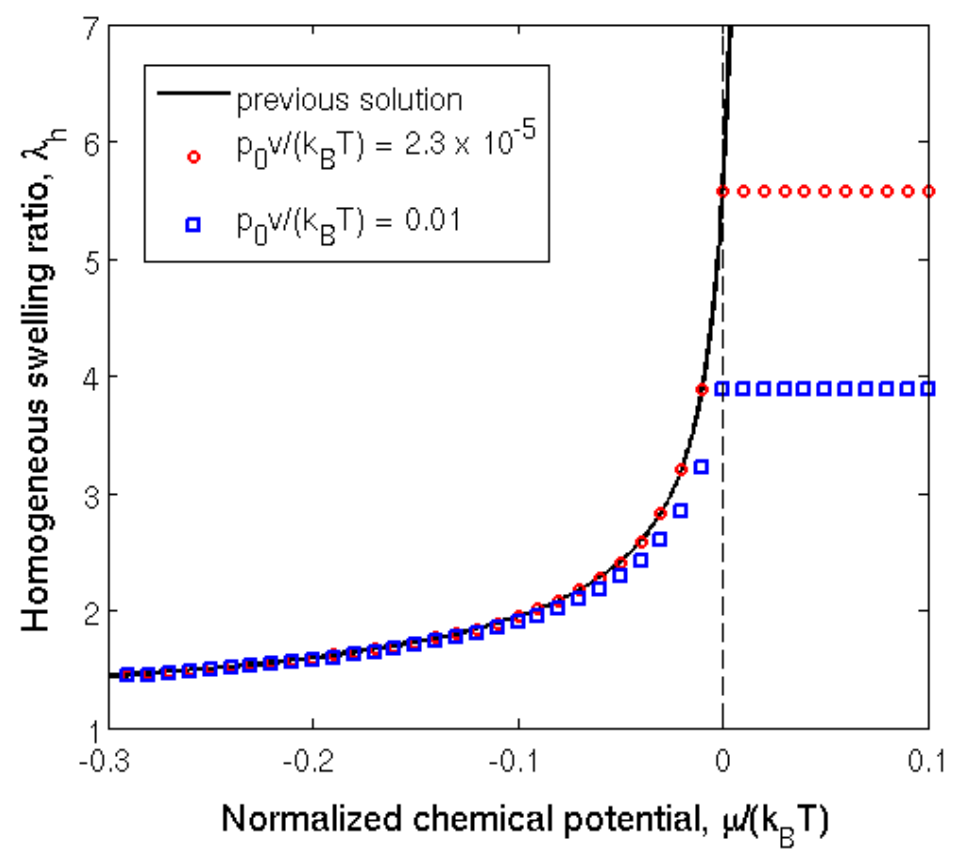

Figure 3. Homogeneous swelling ratio of a hydrogel layer $(N v=0.001$ and $\chi=0.4)$ as a function of the external chemical potential. 

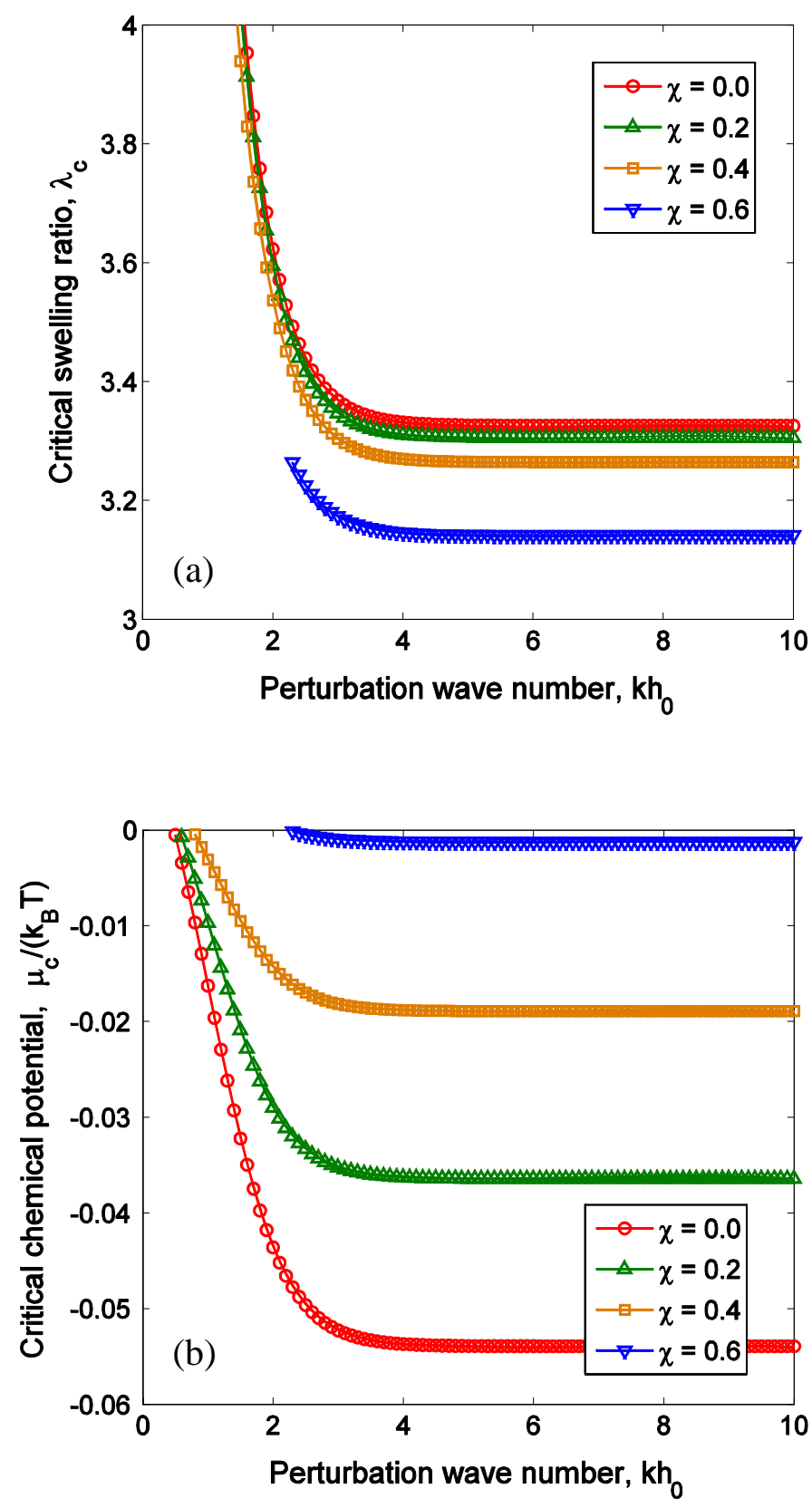

Figure 4. (a) Critical swelling ratio and (b) the corresponding chemical potential, predicted by the linear perturbation analysis, versus the perturbation wave number for $N v=0.001$. 

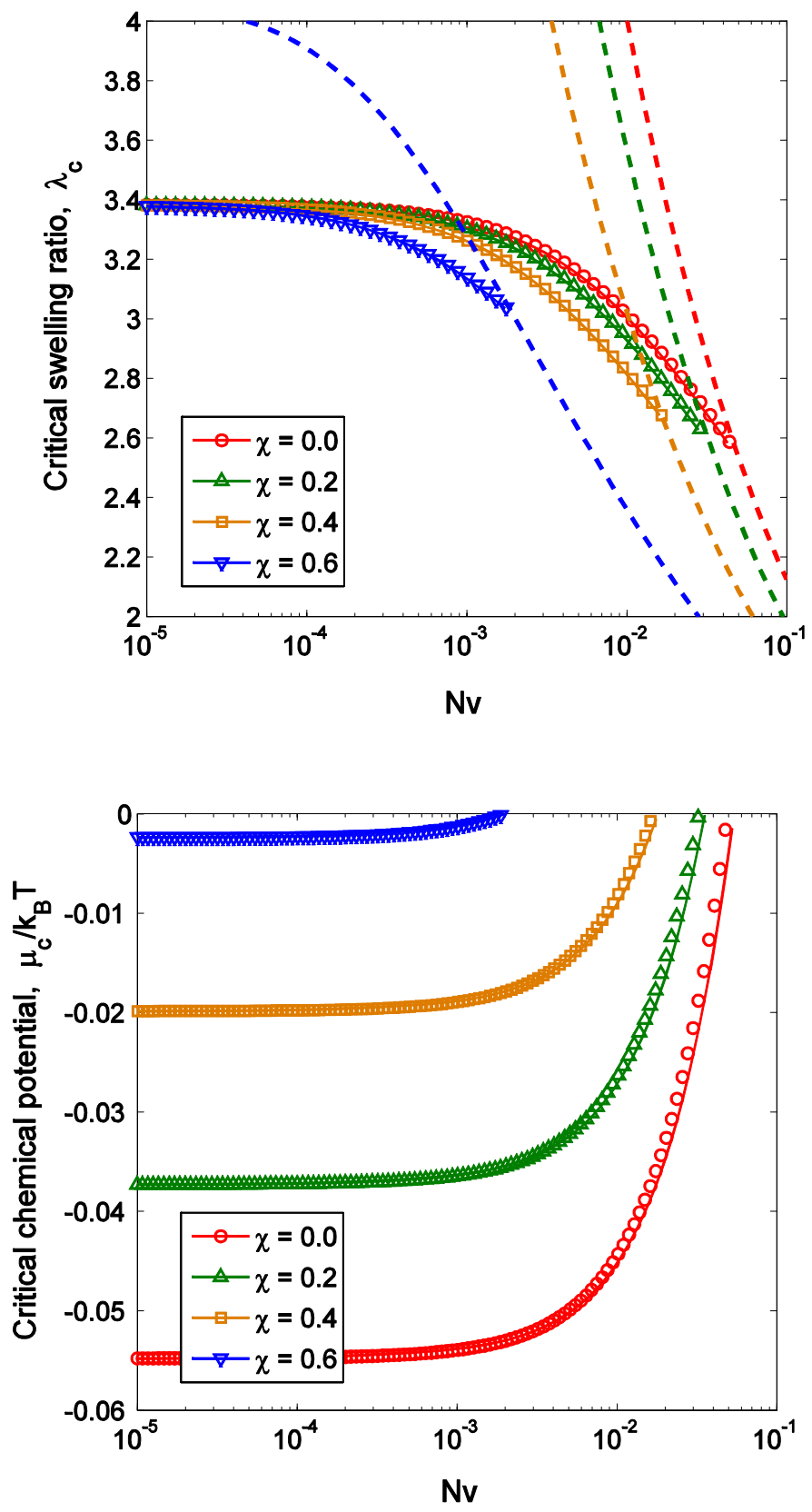

Figure 5. (a) Critical swelling ratio and (b) critical chemical potential, predicted by the linear perturbation analysis at an infinite wave number (short-wavelength limit), versus $N v$ for different values of $\chi$. 


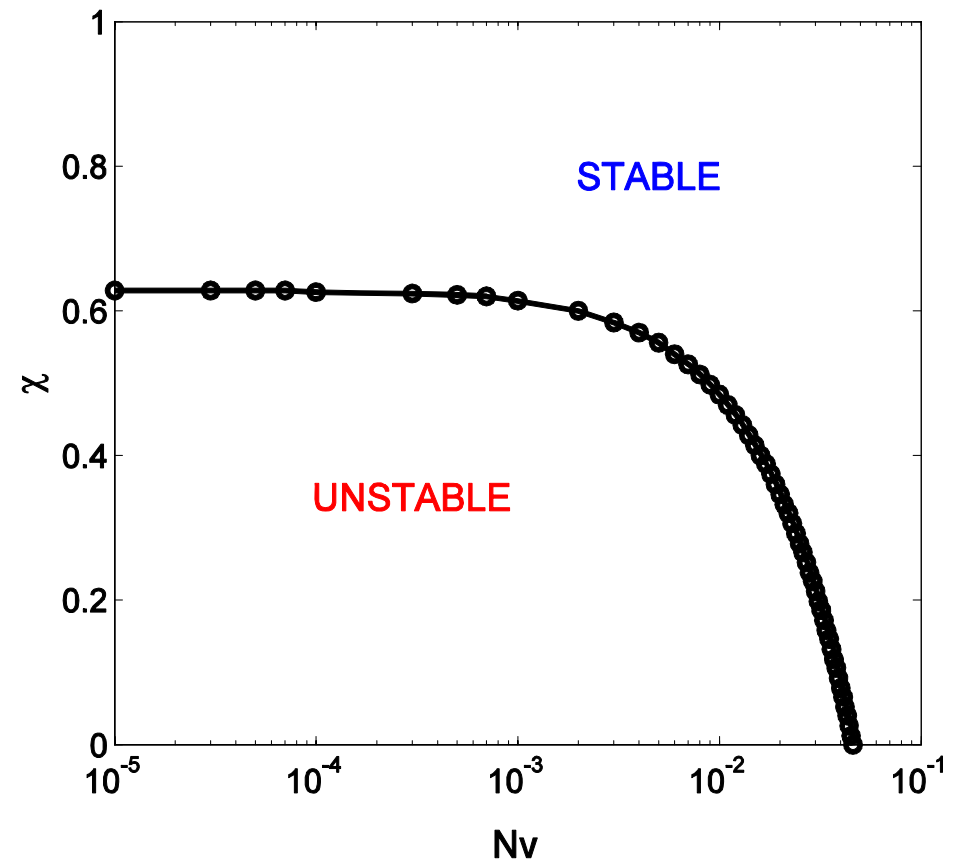

Figure 6. A stability diagram for substrate-confined hydrogel layers. 

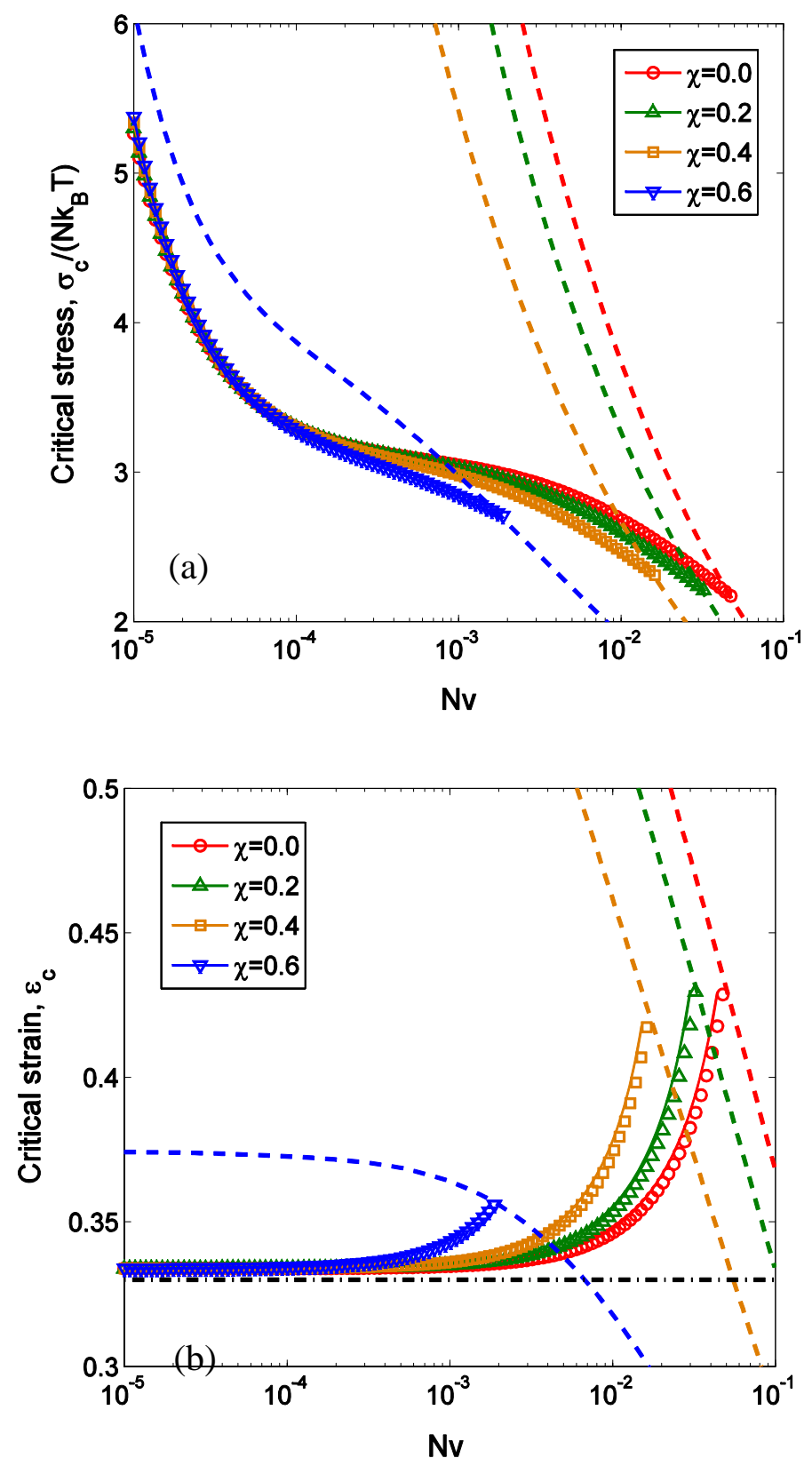

Figure 7. (a) Critical compressive stress and (b) critical linear strain, versus $N v$ for different values of $\chi$. The dashed lines in (a) show the compressive stress at the maximum homogeneous swelling, and the dashed lines in (b) show the effective strain for homogeneous swelling at $\mu=0$. The horizontal dash-dotted line in (b) indicates the critical strain (0.33) for a semi-infinite rubber under equi-biaxial compression. 
(a)

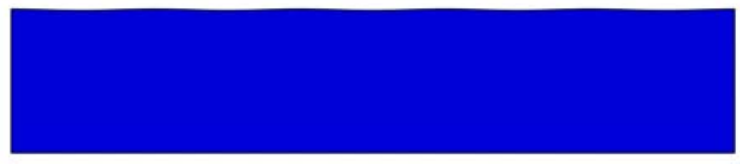

(b)
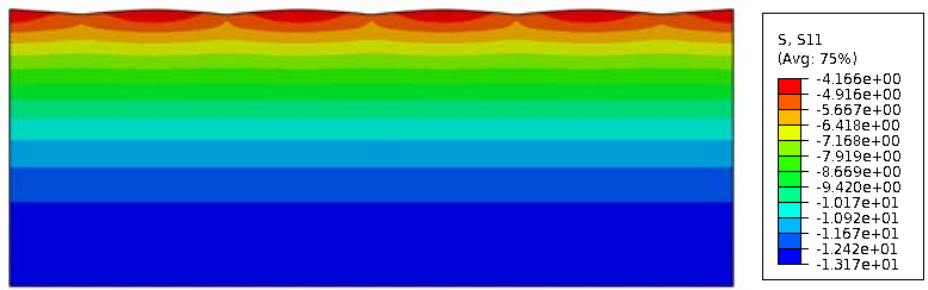

(c)
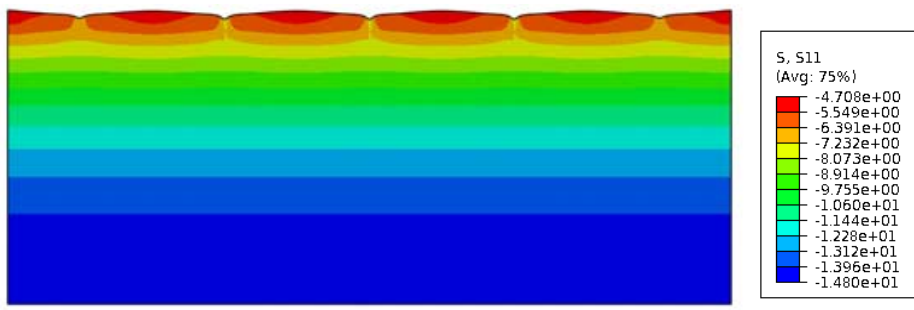

(d)
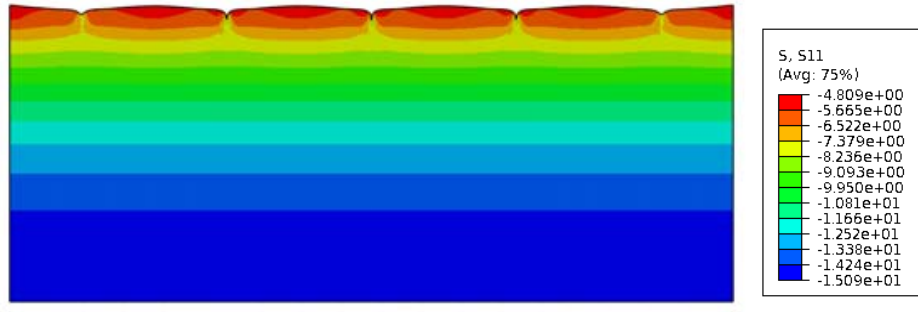

(e)

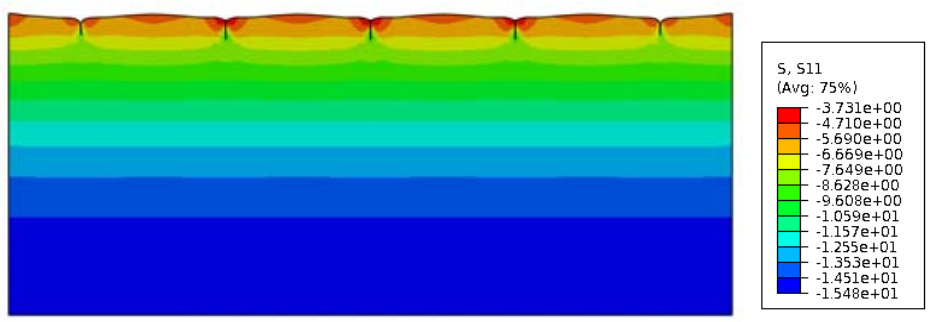

Figure 8. Numerical simulation of swell-induced surface instability of a confined hydrogel layer $(N v=0.001$ and $\chi=0.4)$ on a rigid substrate. Contours show distribution of the compressive true stress in the lateral direction $\left(\sigma_{11}\right)$. (a) Initial perturbation at $\bar{\mu}=-0.0916$; (b) $\bar{\mu}=-0.00456$; (c) $\bar{\mu}=-0.00126$; (d) $\bar{\mu}=-0.000713$; and (e) $\bar{\mu}=0$. The stress magnitude in the scale bar is normalized by the initial shear modulus of the polymer network $\left(N k_{B} T\right)$. 

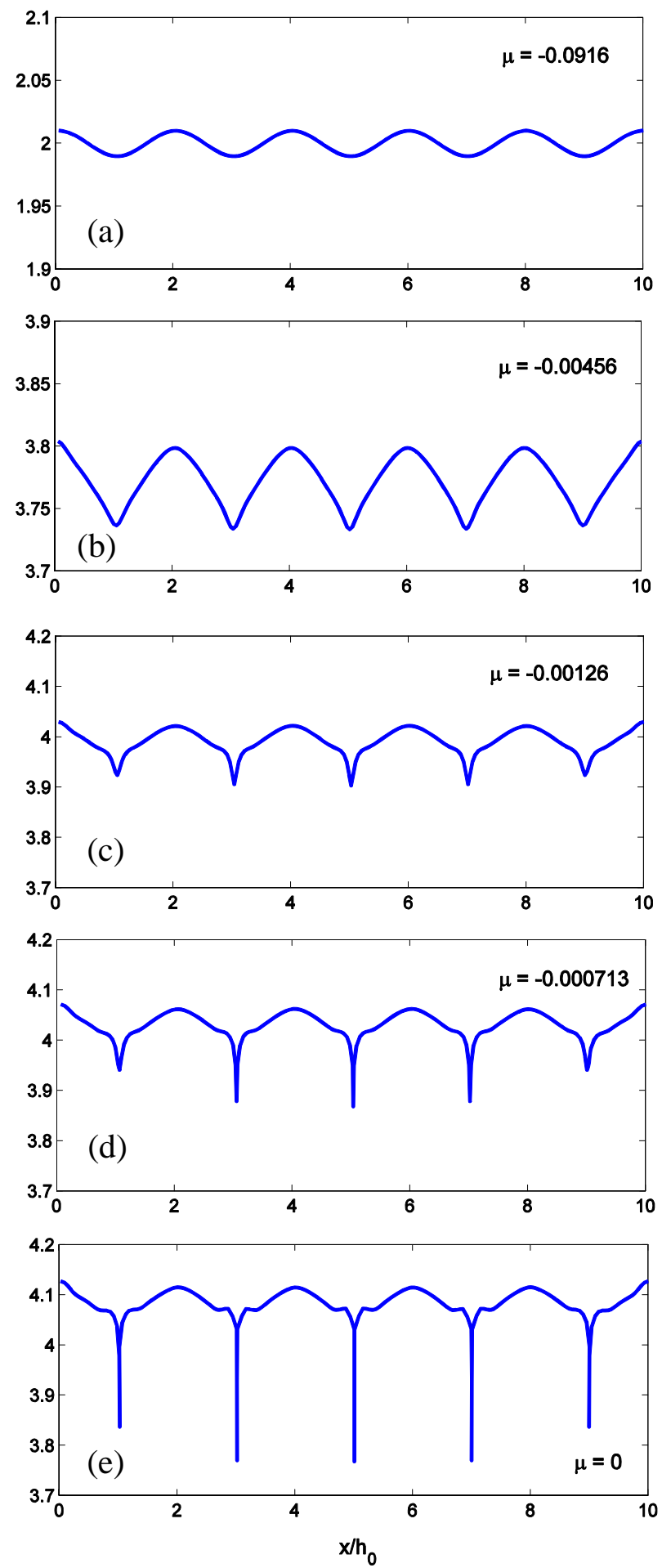

Figure 9. Evolution of the surface profile of a confined hydrogel layer $(N v=0.001$ and $\chi=0.4)$ on a rigid substrate. (a) Initial perturbation at $\bar{\mu}=-0.0916$; (b) $\bar{\mu}=-0.00456$; (c) $\bar{\mu}=$ 0.00126; (d) $\bar{\mu}=-0.000713$; and (e) $\bar{\mu}=0$. 